\title{
Ocorrência e abundância da carcinofauna acompanhante na pesca do camarão sete-barbas, Xiphopenaeus kroyeri Heller (Crustacea, Decapoda), na Armação do Itapocoroy, Penha, Santa Catarina, Brasil
}

\author{
Joaquim Olinto Branco \& Hélio Augusto Alves Fracasso
}

Centro de Ciências Tecnológicas da Terra e do Mar, Universidade do Vale do Itajaí. Caixa Postal 360, 88301 -970 Itajaí, Santa Catarina, Brasil. E-mail: branco@cttmar.univali.br

\begin{abstract}
Occurrence and abundance of carcino-group bycatch in sea-bob shrimp, Xiphopenaeus kroyeri Heller (Crustacea, Decapoda) fishery, in Armação do Itapocoroy, Penha, Santa Catarina, Brazil. The trawl fishery is considered high predatory and damage substantially the bentonic community. The participation of crustaceans in the by-catch of sea-bob shrimp (Xiphopenaeus kroyeri Heller, 1862) is high. The present study aims to analyze the occurrence and the abundance of the group of crustaceans' by-catch of sea-bob shrimp, in the region of Armação do Itapocoroy, Penha, Santa Catarina. The crustaceans were represented by 15 families, 22 genera and 28 species, the higher diversity occurred in 2000-2001 and the lowest in 1997-1998. The contribution of carcinogroup in biomass was superior than $X$. kroyeri in all the samples, except in 2001-2002 when the highest values of sea-bob shrimp were recorded. The six years of samples indicates seasonal variations in the crustaceans group and in spring $(2,4 \pm 1,3 \mathrm{~kg} / \mathrm{h})$ was registered some increase in the biomass reaching the lowest values in winter $(1,8 \pm 1,3 \mathrm{~kg} / \mathrm{h})$.
\end{abstract}

KEY WORDS. Artesian fishery, seasonal fluctuations, bycatch.

RESUMO. A pesca de arrasto é considerada altamente predatória e danifica substancialmente a comunidade bentônica. A participação de crustáceos como fauna acompanhante na pesca artesanal do camarão sete-barbas (Xiphopenaeus kroyeri Heller, 1862) é alta. O objetivo do presente estudo foi analisar a ocorrência e abundância do grupo da carcinofauna na pesca do camarão sete-barbas na Penha, Santa Catarina. Este grupo esteve representado por 15 famílias, 22 gêneros e 28 espécies, com a maior diversidade em 2000-2001 e a menor em 1997-1998. A contribuição em biomassa da carcinofauna foi superior a de $X$. kroyeri em todas as amostragens, menos em 20012002, quando ocorreu a maior taxa de captura da espécie alvo. Uma análise conjunta dos seis anos de coleta indicou uma variação sazonal na biomassa, com os maiores valores na primavera $(2,4 \pm 1,3 \mathrm{~kg} / \mathrm{h})$ e os menores no verão $(1,8 \pm 1,3 \mathrm{~kg} / \mathrm{h})$.

PALAVRAS CHAVE. Fauna acompanhante, flutuações sazonais, pesca artesanal.

A pesca de camarões no Brasil está representada, principalmente, por espécies da família Penaeidae (Severino-Rodrigues et al. 1992). Xiphopenaeus kroyeri (Heller, 1862) é considerado o camarão de maior interesse econômico e o segundo recurso pesqueiro no estado de São Paulo (Fransozo et al. 2000, NAKAgaki \& Negreiros-Fransozo 1998). Em Santa Catarina, a frota industrial de arrasto duplo desembarcou um total de 4.242t de crustáceos no ano de 2000, destes o camarão sete-barbas contribuiu com 0,305 t, sendo superado pelo camarão barba-ruça (Artemesia longinaris Bate, 1888) e santana (Pleoticus muelleri (Bate, 1888)), 2.466 e 1.068t, respectivamente (Gер/СtтмAR 2000).

A pesca de arrasto com redes equipadas com portas é considerada predatória danificando substancialmente as comu- nidades bentônicas, sendo freqüentemente realizada em criadouros de espécies com interesse econômico. O conhecimento de como essa modalidade atua sobre os recursos marinhos é fundamental para a sustentabilidade deste ecossistema (RufFino \& CAstello 1992).

A participação dos crustáceos decápodes na composição da fauna associada à pesca de arrasto é elevada, superando consideravelmente a biomassa de camarões em condições de comercialização (Coelho et al. 1986). Muitas espécies que são capturadas ao acaso, apresentam pequeno porte e tradicionalmente não possuem valor comercial, sendo descartadas pelos pescadores artesanais. Porém, no futuro, podem despertar interesse e adquirir prestígio no comércio (MANTELATTO et al. 1995). 
A exploração comercial de siris e caranguejos é um fator importante na economia de alguns países americanos, europeus e no Japão. Os Portunídeos do gênero Callinectes, suportam importantes pescarias no Atlântico, incluindo a costa ao sul dos Estados Unidos e Golfo do México (Roman-Contreras 1986).

No Brasil, esta cultura alimentícia ainda se restringe a poucas espécies, e o baixo valor comercial de braquiúros que são capturados acidentalmente nas pescas de arrasto de fundo, gera grande desperdício (Pereira-Barros 1981, Petti 1997). Neste sentido, teve-se como objetivo analisar a ocorrência e abundância da carcinofauna acompanhante do camarão sete-barbas, na região da Armação do Itapocoroy, Penha, Santa Catarina.

\section{MATERIAL E MÉTODOS}

A pesca artesanal do camarão sete-barbas na Armação do Itapocoroy $\left(26^{\circ} 40^{\prime}-26^{\circ} 47^{\prime} \mathrm{S}\right.$ e $\left.48^{\circ} 36^{\prime}-48^{\circ} 38^{\prime} \mathrm{W}\right)$ é denominada "pesca de sol a sol", com início das atividades ao amanhecer e encerramento antes do por do sol (Branco et al. 2001). Nessa arte de pesca são utilizadas baleeiras entre 7,0 e 10,0 m de comprimento, com 2,0 a 3,0 m de boca, motor variando entre $16 \mathrm{e}$ 40 Hp e não possui reservatórios específicos para armazenar e manter refrigerado o pescado.

Durante o período de agosto/1996 a julho/2002 foram realizadas coletas mensais em três áreas tradicionais de atuação da pesca artesanal com profundidade variando entre 5,0 a 20,0 m: Ponta da Vigia, Ilha Feia e Ilhas Itacolomis, com duração de uma hora. Foram utilizadas duas redes-de-arrastos com portas, malha de $3,0 \mathrm{~cm}$ na manga e corpo e de $2,0 \mathrm{~cm}$ no ensacador, tracionada por baleeira com velocidade média de 2,0 nós. Sendo que a área amostrada em cada arrasto foi de aproximadamente $1.852 \mathrm{~m}^{2}$, totalizando ao final das três áreas, $5.556 \mathrm{~m}^{2}$ (Branco \& Lunardon-Branco 1993).

O material capturado nos arrastos dirigido ao camarão sete-barbas foi acondicionado em caixa de isopor e resfriados com gelo.

No laboratório, a identificação dos componentes da fauna acompanhante foi realizada de acordo com a bibliografia específica, sendo registrado nas coletas, o peso e o número de exemplares. Nesta etapa, a espécie alvo X. kroyeri não foi computada como carcinofauna, mas teve seu peso e número de exemplares determinado para posterior comparação com a mesma.

O termo carcinofauna aqui utilizado, segue a mesma denominação dos trabalhos de fauna acompanhante de camarões, abrangendo os crustáceos decápodes exceto a espécie-alvo (Severino-Rodrigues et al. 2002, Clucas 1997). Assim, os crustáceos capturados foram identificados (Pérez-Farfante 1978, Melo 1996), registrando o número e o peso por amostra. De acordo com a ocorrência nas coletas ao longo do ano, as espécies foram classificadas em três categorias: regular ( 9 a 12 meses); sazonal (6 a 8 meses) e ocasional (um a cinco meses) (ANsAri et al. 1995).

Os demais grupos da fauna acompanhante (Ictiofauna, Cnidofauna, Equinodermofauna e Malacofauna) foram objetos de outros trabalhos. Neste estudo, o material inorgânico (plástico, lata e ferro) e orgânico (algas, conchas e outros) foram em conjunto pesados e considerados como lixo.

A captura por unidade de esforço (CPUE) em kg/hora foi calculada mensalmente. Embora com pequenas variações entre as áreas amostradas, os dados foram reunimos e apresentados como a média por arrasto.

A partir do somatório das CPUE da fauna acompanhante nos seis anos de coleta, foi determinada a participação relativa da carcinofauna em relação aos demais grupos integrantes da pesca do camarão sete-barbas.

Após testada a homogeneidade das médias foi utilizada a ANOVA (SOKAL \& RoHLF 1969) para comparar as flutuações da CPUE da carcinofauna por mês, sazonal e ano. O contraste das médias por meio do teste Tuckey-Kramer, foi aplicado na ocorrência de diferenças significativas para indicar a fonte causadora das variações.

\section{RESULTADOS}

A carcinofauna esteve representada por 15 famílias, 22 gêneros e 28 espécies, sendo que a maior diversidade específica ocorreu no período de 2000-2001 e a menor em 1997-1998 (Tab. I). Destas espécies, 13 foram comuns entre os anos de amostragens; no total geral, $64,2 \%$ foi de ocorrência ocasional, $19,5 \%$ sazonal e 16,3\% regular. As famílias com maior diversidade, Portunidae e Penaeidae em conjunto, contribuíram com $32,1 \%$ das espécies e $49,0 \%$ do total de exemplares capturados, e as menores (uma espécie) representaram 35,7\% e 49,1\%, respectivamente (Tab. I).

A figura 1 indica que a participação relativa em biomassa da carcinofauna foi de $16,4 \%$, sendo inferior a ictiofauna $(38,7 \%)$ e cnidofauna $(18,9 \%)$, sendo superior a espécie-alvo, X. kroyeri $(11,4 \%)$, lixo $(8,7 \%)$, malacofauna $(4,2 \%)$ e equinodermofauna $(1,8 \%)$.

A contribuição média anual da carcinofauna em biomassa foi superior a do camarão sete-barbas em todo período, exceto no ano de 2001-2002 onde ocorreram as maiores CPUE médias da espécie-alvo e as menores do grupo acompanhante (Fig. 2). A partir de 1996/1997 foram registrados incrementos graduais nas médias da carcinofauna até 1998/1999, reduzindo gradualmente até 2001/2002; enquanto que as médias do camarão oscilaram ao longo do estudo (Fig. 2).

As figuras 3 a 8 mostram que ocorreram flutuações sazonais das CPUE da carcinofauna com diferença significativa $\left(\mathrm{F}_{5-66}=3,794 ; \mathrm{p}=0,004\right)$ entre os anos estudados. O contraste das médias indicou que essa diferença foi ocasionada pelas discrepâncias nas taxas de captura em 1998-1999 e 2000-2001 e entre 1998-1999 e 2001-2002.

A partir de setembro de 1996 ocorreu um incremento gradual da captura até março $(3,3 \pm 3,0 \mathrm{~kg})$, oscilando até atingir as menores CPUE em julho $(0,8 \pm 0,6$ kg) (Fig. 3). Em 19971998 ocorreram maiores taxas de captura e oscilações em relação ao ano anterior, com pico em dezembro $(4,1 \pm 2,5 \mathrm{~kg})$ e as menores em julho $(0,8 \pm 0,4 \mathrm{~kg})$ (Fig. 4). 

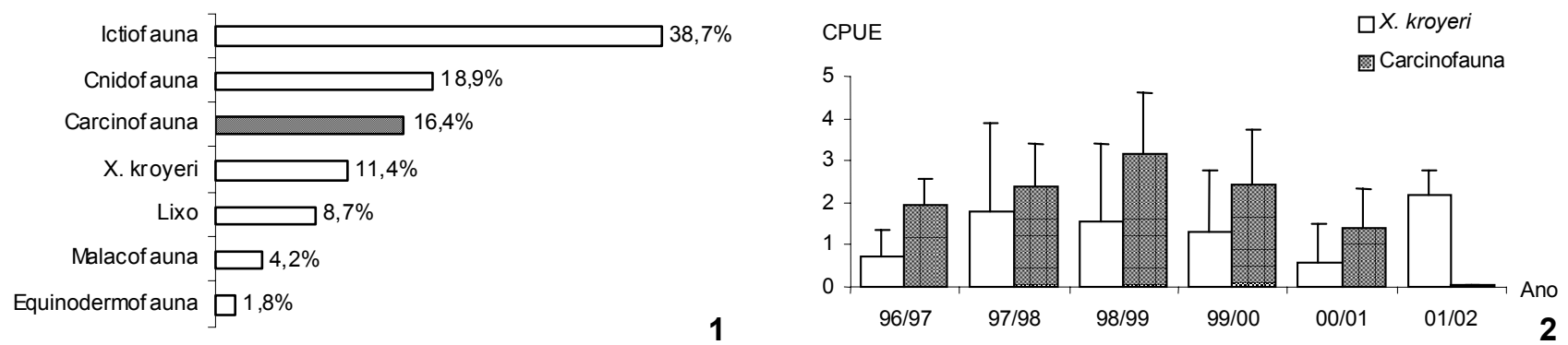

Figuras 1-2. (1) Participação relativa em biomassa dos grupos acompanhantes na pesca artesanal do camarão sete-barbas, nos anos de agosto/1996 a julho/2002; (2) Participação da carcinofauna acompanhante e da espécie-alvo (X. kroyeri) em CPUE kg/h (médio), ao longo do período de amostragem. As barras verticais são referentes aos desvios padrões.
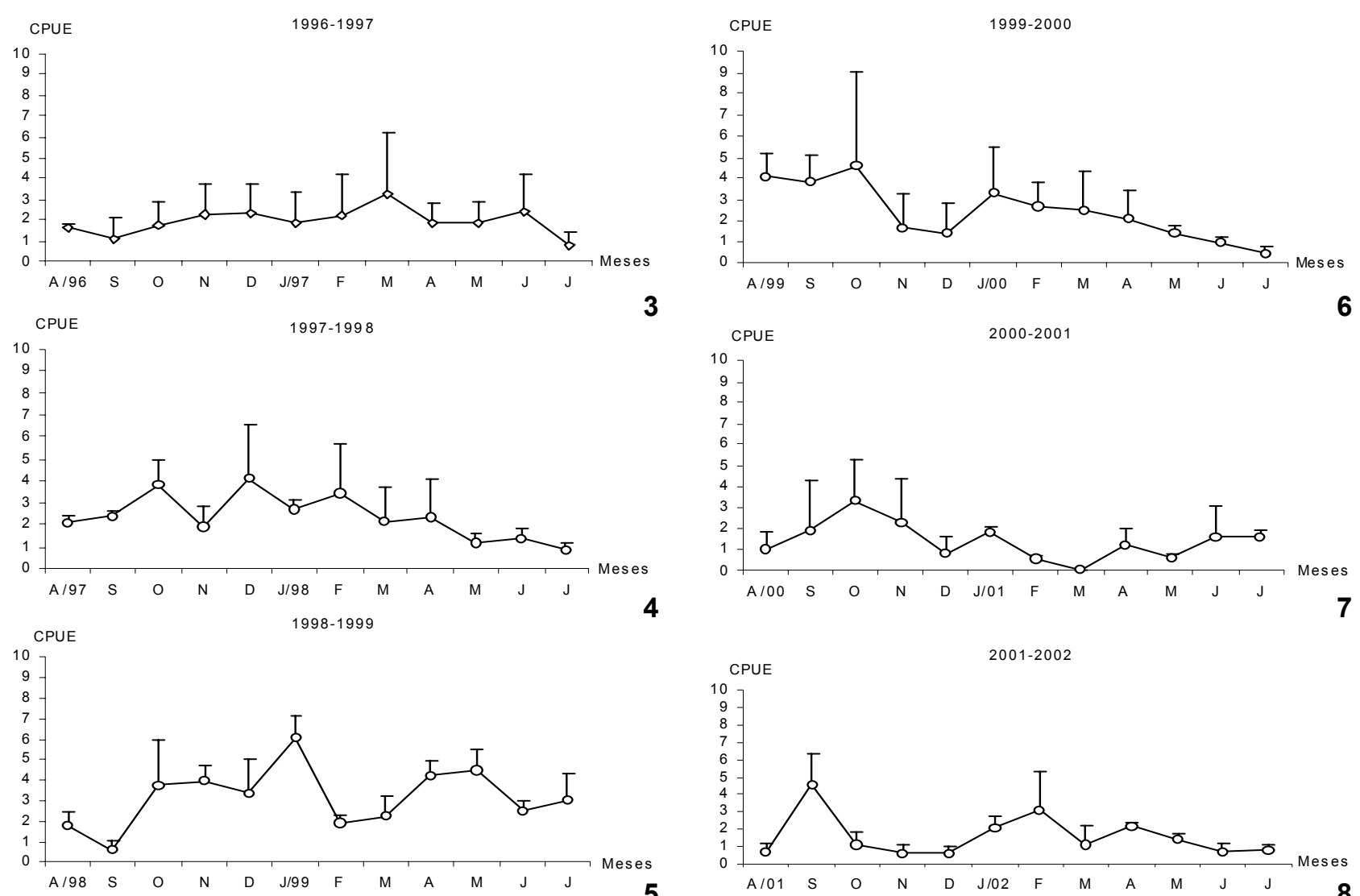

Figuras 3-8. Variação mensal da CPUE(kg/h) média dos crustáceos decápodes, durante o período de agosto/1996 a julho/2002. As barras verticais são referentes aos desvios padrões.

No ano de 1998-1999 as menores CPUE ocorreram entre agosto e setembro com incremento gradual até janeiro $(6,1 \pm$ $1,1 \mathrm{~kg}$ ), seguido de redução e oscilação na biomassa até julho (3,0 $\pm 1,3 \mathrm{~kg}$ ) (Fig. 5). Em 1999-2000 as maiores CPUE médias foram registradas de agosto a outubro, oscilando até janeiro, seguido de decréscimo gradual atingindo as menores taxas de captura em julho $(0,4 \pm 0,3 \mathrm{~kg})$ (Fig. 6).

No período de 2000-2001 e 2001-2002 a flutuação da
CPUE seguiu a mesma tendência dos anos anteriores, com oscilações até dezembro seguido de acréscimo e variações moderadas até julho (Figs 7 e 8).

Embora a ANOVA $\left(\mathrm{F}_{5-18}=3,373 ; \mathrm{p}=0,025\right)$ tenha indicado diferença significativa entre as CPUE médias da carcinofauna e as estações do ano; numa análise geral, a figura 9 mostra uma redução gradativa das capturas a partir da primavera $(2,4 \pm 1,3 \mathrm{~kg})$ até atingir os menores valores nos meses de inverno $(1,8 \pm 1,3 \mathrm{~kg})$.

Revista Brasileira de Zoologia 21 (2): 295-301, junho 2004 


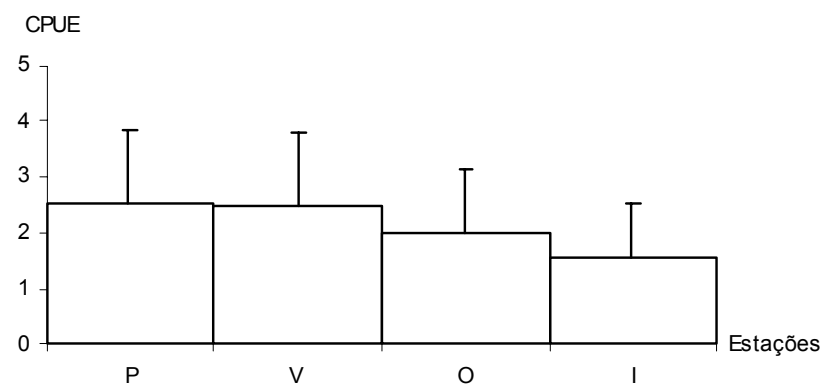

Figura 9. Flutuação sazonal da CPUE $(\mathrm{kg} / \mathrm{h})$ média, entre os anos de agosto/1996 a julho/2002. As barras verticais são referentes aos desvios padrões $(\mathrm{P}=2,53 ; \mathrm{V}=2,48 ; \mathrm{O}=2,00 \mathrm{e} \mathrm{I}=1,57)$.

\section{DISCUSSÃO}

A fauna acompanhante da pesca dirigida ao camarão setebarbas apresenta uma multiplicidade faunística considerável, representada, em sua maioria, por indivíduos imaturos, sendo desconhecido o impacto causado pela mortalidade desta fauna no equilíbrio ecológico das áreas de pesca (SEverino-Rodrigues et al. 1985), contribuindo para uma perda valiosa de alimento e biodiversidade. Assim, a sustentabilidade das operações pesqueiras mundiais é preocupante, não pela captura da espéciealvo ou daquelas protegidas pela opinião pública, mas pelo descarte das espécies que podem, no futuro, serem usadas como fonte de alimento (CluCAS 1997).

Tabela I. Relação das espécies integrantes da carcinofauna acompanhante na pesca dirigida ao camarão sete-barbas, no período de agosto/1996 a julho/2001. A ocorrência das espécies está representada por: (>) regular, (+) sazonal e (<) ocasional. (N) Somatório das três áreas de arrastos.

Táxon $\quad \frac{1996-1997}{\mathrm{~N} \text { Ocor. }} \frac{1997-1998}{\mathrm{~N} \text { Ocor. }} \frac{1998-1999}{\mathrm{~N} \text { Ocor. }} \frac{1999-2000}{\mathrm{~N} \text { Ocor. }} \frac{2000-2001}{\mathrm{~N} \text { Ocor. }} \frac{2001-2002}{\mathrm{~N} \text { Ocor. }}$

\begin{tabular}{|c|c|c|c|c|c|c|c|c|c|c|c|c|}
\hline \multicolumn{13}{|l|}{ Penaeidae } \\
\hline Farfantepenaeus brasiliensis Latreille, 1817 & 38 & $<$ & - & - & - & - & - & - & 50 & $<$ & - & - \\
\hline Farfantepenaeus paulensis (Pérez Farfante, 1967) & 8 & $<$ & - & - & 99 & + & 2 & $<$ & 8 & $<$ & 14 & $<$ \\
\hline Litopenaeus schmitti (Burkenroad, 1936) & 41 & + & 83 & $>$ & 38 & + & 23 & $<$ & 35 & + & 60 & + \\
\hline Trachypenaeus constrictus (Stimpson, 1874) & 59 & $<$ & - & - & - & - & - & - & - & - & - & - \\
\hline Artemesia longinaris Bate, 1888 & 2984 & $<$ & 1272 & $<$ & 1982 & $>$ & 4772 & + & 3038 & + & 713 & $<$ \\
\hline \multicolumn{13}{|l|}{ Lismatidae } \\
\hline Exhippolysmata oploforoides Holthuis, 1948 & 16 & $<$ & 13 & $<$ & 22 & + & 69 & $<$ & 43 & $<$ & 111 & + \\
\hline \multicolumn{13}{|l|}{ Sergestidae } \\
\hline Acetes americanus americanus Ortmann, 1893 & 2800 & $>$ & 621 & $<$ & 1916 & $>$ & 368 & $<$ & 6536 & $<$ & 20 & $<$ \\
\hline \multicolumn{13}{|l|}{ Solenoceridae } \\
\hline Pleoticus muelleri (Bate, 1888) & 396 & $<$ & 1965 & $<$ & 1286 & $>$ & 2364 & + & 1413 & + & 736 & + \\
\hline \multicolumn{13}{|l|}{ Sicyoniidae } \\
\hline Sycione dorsalis (Bate, 1878) & 79 & $<$ & 245 & $<$ & 166 & $>$ & 170 & + & 75 & + & 23 & $<$ \\
\hline \multicolumn{13}{|l|}{ Alpheidae } \\
\hline Alpheus & - & - & 4 & $<$ & - & - & 4 & $<$ & 3 & $<$ & 16 & $<$ \\
\hline \multicolumn{13}{|l|}{ Diogenidae } \\
\hline Dardanus insignis (de Sansure, 1858) & 1 & $<$ & 22 & $<$ & 31 & + & 28 & $<$ & 66 & $<$ & 6 & $<$ \\
\hline \multicolumn{13}{|l|}{ Squillidae } \\
\hline Squilla grenadensis Manning, 1969 & - & - & 14 & $<$ & 12 & $<$ & 20 & $<$ & 8 & $<$ & 96 & $<$ \\
\hline \multicolumn{13}{|l|}{ Paguridae } \\
\hline Loxopagurus loxochelis (Moreira, 1901) & - & - & 24 & $<$ & 20 & $<$ & 8 & $<$ & 44 & $<$ & 31 & $<$ \\
\hline \multicolumn{13}{|l|}{ Calappidae } \\
\hline Hepatus pudibundus (Herbst, 1785) & 221 & + & 961 & $>$ & 1380 & $>$ & 872 & $>$ & 542 & $>$ & 706 & $>$ \\
\hline \multicolumn{13}{|l|}{ Porcellanidae } \\
\hline Porcellana sayana (Leach, 1820) & - & - & - & - & - & - & - & - & - & - & 3 & $<$ \\
\hline & & & & & & & & & & & Cor & tinua \\
\hline
\end{tabular}

Revista Brasileira de Zoologia 21 (2): 295-301, junho 2004 
Tabela I. Continuação.

\begin{tabular}{|c|c|c|c|c|c|c|c|c|c|c|c|c|}
\hline \multirow{2}{*}{ Táxon } & \multicolumn{2}{|c|}{ 1996-1997 } & \multicolumn{2}{|c|}{ 1997-1998 } & \multicolumn{2}{|c|}{ 1998-1999 } & \multicolumn{2}{|c|}{$1999-2000$} & \multicolumn{2}{|c|}{ 2000-2001 } & \multicolumn{2}{|c|}{ 2001-2002 } \\
\hline & $\mathrm{N}$ & Ocor. & $\mathrm{N}$ & Ocor. & $\mathrm{N}$ & Ocor. & $\mathrm{N}$ & Ocor. & $\mathrm{N}$ & Ocor. & $\mathrm{N}$ & Ocor. \\
\hline \multicolumn{13}{|l|}{ Leucossidae } \\
\hline Persephona mediterranea (Herbst, 1794) & 78 & $<$ & 122 & $<$ & 28 & + & 4 & $<$ & 12 & $<$ & 22 & $<$ \\
\hline Persephona punctata (Linnaeus, 1758) & 19 & $<$ & 20 & $<$ & 102 & $>$ & 124 & + & 22 & $<$ & 153 & + \\
\hline Persephona lichtensteinii Leach, 1817 & - & - & - & - & - & - & - & - & - & - & 53 & $<$ \\
\hline \multicolumn{13}{|l|}{ Majidae } \\
\hline Libinia ferreirae Brito Capello, 1871 & 17 & $<$ & - & - & - & - & - & - & 2 & $<$ & - & - \\
\hline Libinia spinosa $(\mathrm{H}$. Milne Edwards, 1834) & 26 & $<$ & 8 & $<$ & 43 & $>$ & 56 & + & 66 & + & 53 & + \\
\hline \multicolumn{13}{|l|}{ Portunidae } \\
\hline Callinectes danae (Smith, 1869) & 469 & $>$ & 453 & $>$ & 182 & $<$ & 174 & $>$ & 206 & + & 123 & + \\
\hline Callinectes ornatus (Ordway, 1863) & 1183 & $>$ & 2233 & $>$ & 1935 & $<$ & 2280 & $>$ & 728 & + & 1055 & $>$ \\
\hline Callinectes sapidus Rathbun, 1896 & 2 & $<$ & - & - & - & - & 4 & $<$ & 6 & $<$ & - & - \\
\hline Portunus spinimanus (Latreille, 1819) & 1 & $<$ & - & - & 3 & $<$ & 14 & $<$ & 46 & $<$ & 16 & $<$ \\
\hline Cronius ruber (Lamarck, 1818) & 1 & $<$ & 16 & $<$ & - & - & - & - & 2 & $<$ & - & - \\
\hline Arenaeus cribarius (Lamarck, 1818) & 12 & $<$ & 2 & $<$ & 8 & $<$ & - & - & 26 & $<$ & 1 & $<$ \\
\hline \multicolumn{13}{|l|}{ Xanthidae } \\
\hline Pilumnus dasypodus Kingsley, 1879 & - & - & - & - & 4 & $<$ & 3 & $<$ & - & - & - & - \\
\hline Menipe nodifrons Stimpson, 1859 & 1 & $<$ & - & - & 1 & $<$ & - & - & - & - & - & - \\
\hline Total & 8452 & & 8078 & & 9258 & & 11359 & & 12977 & & 4011 & \\
\hline
\end{tabular}

Apesar da diversidade e importância na rede trófica das áreas pesqueiras, existem poucas informações recentes disponíveis no litoral brasileiro que analisem em conjunto esses grupos na pesca de arrasto dirigida aos camarões (Clucas 1997, Severino-Rodrigues et al. 2002, GraÇa-Lopes et al. 2002). Entretanto, em estudo da megafauna bêntica do litoral norte de São Paulo, os Crustacea foram o grupo dominante, seguido pelos Molusca e Equinodermata (PIREs-VANin et al. 1995).

Aspectos biológicos e pesqueiros da espécie-alvo X. kroyeri no litoral brasileiro recebeu atenção especial, no Nordeste (MотAAlves \& Rodrigues 1977, Coelho \& Santos 1993), em São Paulo (Vieira 1947, Neiva \& Wise 1963, Santos et al. 1969, SeverinoRodrigues et al. 1992, Nakagaki \& Negreiros-Fransozo 1998, Fransozo et al. 2000), no Paraná (BRANCo et al. 1994) e em Santa Catarina (Nascimento \& Poli 1986, Branco et al. 1999) contribuindo com informações relevantes para o estudo desta espécie.

Em geral, a composição percentual dos grupos taxonômicos de espécies que integram a fauna acompanhante de camarões, pode variar em função da área de pesca, profundidade e época do ano (Carranza-Fraser \& Grande 1982, Ruffino \& Castello 1992). Entretanto, independentemente do local de atuação da pesca na Armação do Itapocoroy, a Ictiofauna foi o principal grupo capturado, seguido da Cnidofauna, Carcinofauna, Malacofauna e Equinodermofauna.

A participação dos crustáceos decápodes nos descartes da pesca de arrasto com portas é considerada alta (Hill \& WASSENBERG 2000). As informações disponíveis realçam esta situação, sendo que na Enseada do Mucuripe, Ceará, os camarões Penaeidae contribuíram com a maior diversidade e abundância seguida dos Brachyura com 18 espécies (Sampaio \& Fausto-Filho 1984). Enquanto que em Ubatuba, São Paulo os braquiúros participaram com 50 espécies distribuídas em 10 famílias (MantelatTo \& Fransozo 2000), na desembocadura da Praia de Perequê, Guarujá, São Paulo com 20 famílias e 41 espécies (Severino-Rodrigues et al. 2002). Já em Matinhos, litoral do Paraná, a carcinofauna esteve representada por 11 espécies pertencentes a quatro famílias (Branco \& LunARdon-Branco 1993); e na Lagoa do Peixe, Rio Grande do Sul, com a utilização da rede tipo "coca" foi registrada a ocorrência de 11 espécies de decápodes (SANTOS et al. 2000). No presente estudo, a carcinofauna esteve representada por 28 espécies distribuídas em 15 famílias.

Portunidae apresentou maior abundância no litoral brasileiro, com 80,9\% no Ceará (Sampaio \& Fausto-Filho 1984), 84,2\% em São Paulo (Mantelatto \& Fransozo 2000), 86,9\% no Paraná (Lunardon-Branco \& Branco 1993), e 93,2\% em Santa Catarina (BRANCo et al. 1998). Neste estudo, ocorreu um decréscimo na participação dos portunídeos $(67,9 \%)$, contrariando a tendência observada de incremento gradual dessa família em direção ao sul da costa brasileira. As espécies Callinectes ornatus Ordway, 1863, C. danae Smith, 1869 e H. pudibundus (Herbst, 1785) 
foram as dominantes em todo o litoral.

As maiores taxas de captura da carcinofauna na Armação do Itapocoroy foram observadas nos meses de primavera, corroborando com os resultados obtidos por Branco et al. (2002) e SANTos et al. (2000). Este padrão sazonal na abundância da carcinofauna pode ser atribuído em parte, pelas características hidrográficas da região (MATsuURA 1986, Boschi 1969), aos eventos dos ciclos de vida característicos destes organismos (NAKAGAKI et al. 1995), com a temperatura local (D’INCAO 1984), além da profundidade e granulometria do sedimento (SANTOS \& CoELHO 1996).

Porções consideráveis de crustáceos que são subutilizados nos locais de pesca com arrasto voltada para captura de camarões, poderiam movimentar a economia local ou até mesmo regional, visto que a produção média para a colônia de Bebedouro (AL), no período de 1967 a 1971 foi de 312,5 toneladas com rendimento de 112,5 toneladas de carne para comercialização (Pereira-Barros \& Travassos 1972). Para os caranguejos de manguezais, Cardisoma guanhumi Latreille, 1852 apresentou um rendimento médio da carne em torno de $18 \%$ e Menipe nodifrons Stimpson, 1859 de 21,5\% (Oshiro et al. 1999).

Atualmente, o preço de mercado que poderia ser atingido pela carcinofauna acompanhante não viabiliza os custos de manutenção a bordo, transporte, desembarque e comercialização. Caso as embarcações que atuam na pesca artesanal do camarão sete-barbas apresentassem maior capacidade de refrigeração da produção, esta poderia ser aproveitada com base nas tendências de mercado, sendo comercializada in-natura ou processada.

\section{REFERÊNCIAS BIBLIOGRÁFICAS}

Ansari, Z.A.; A. Chatterfi; B.S. Ingole; R.A. SREePada; C.U. RivonKar, $\&$ A.H. Parulenkar. 1995. Community structure and sazonal variation of on inshore demersal fish community at Goa, west coast of India. Estuarine Coastal and Shelf Science, London, 41: 593-610.

Branco, J.O. \& M.J. Lunardon-Branco. 1993. Crescimento e tamanho de primeira maturação em Callinectes ornatus Ordway, 1863 (Decapoda, Portunidae) na Região de Matinhos, Paraná, Brasil. Arquivos de Biologia e Tecnologia, Curitiba, 36 (3): 497-503.

Branco, J.O.; M.J. Lunardon-Branco \& A. de Fenis. 1994. Crescimento de Xiphopenaeus kroyeri (Heller, 1862) (Crustacea: Natantia: Penaeidae) da Região de Matinhos, Paraná, Brasil. Arquivos de Biologia e Tecnologia, Curitiba, 37 (1): 1-8.

Branco, J.O.; M.J. Lunardon-Branco; A.C. Peret; F.X. Souto; R. Schveitzer \& W.G. Vale. 1998. Associações entre macroinvertebrados e peixes demersais na Armação do Itapocoroy, Penha, SC, Brasil. Brazilian Archives of Biology and Technology, Curitiba, 41 (2): 268-277.

Branco, J.O.; M.J. Lunardon-Branco; F.X. Souto \& C.R. Guerra. 1999. Estrutura populacional do camarão sete-barbas Xiphopenaeus kroyeri (Heller, 1862), na foz do rio Itajaí-Açú, Itajaí, SC, Brasil. Brazilian Archives of Biology and Technology, Curitiba, 42 (1): 115-126.
Branco, J.O.; J.R.R. BraUn \& J.R. Verani. 2001. Seasonal variation in the abundance of seabirds in areas of mariculture. Brazilian Archives of Biology and Technology, Curitiba, 44 (4): 395-399.

Branco, J.O.; M.J. Lunardon-Branco \& F.X. Souto. 2002. Estrutura populacional de Portunus spinimanus Latreille (Crustacea, Portunidae) na Armação do Itapocoroy, Penha, Santa Catarina, Brasil. Revista Brasileira de Zoologia, Curitiba, 19 (3): 731-738.

Boschi, E.E. 1969. Estudo biológico pesquero del camarón Artemesia longinaris Bate, de Mar del Plata.

Boletin de la Biologia Marina, Mar Del Plata, Argentina, 18: $1-47$.

Carranza-Fraser, J. \& J.M. Grande. 1982. Experiencia de Mexico en el aprovechamienro de la fauna de acompañaminto del camarón. Process of Gulf Caribbean Fisheries Institute, Miami, 39: 109-111.

CLuCAS, I. 1997. A study of the options for utilization of bycatch and discards from marine capture fisheries. FAO, Rome, 59p.

Coelho, P.A. \& M.C.F. SANTos. 1993. Época da reprodução do camarão sete-barbas, (Xiphopenaeus kroyeri) (Heller, 1862) (Crustacea,Decapoda, Penaeidae) na região de Tamandaré, PE. Técnico Científico CEPENE, Tamandaré, 1 (1): 171-186.

Coelho, J.A.P.; A. Puzzi; R. Graça-Lopez; E.S. Rodrigues \& O. PreTO JR. 1986. Análise da rejeição de peixes na pesca artesanal dirigida ao camarão sete-barbas (Xiphopenaeus kroyeri) no Litoral de São Paulo. Boletim do Instituto da Pesca, São Paulo, 13 (2): 51-61.

D'Incao, F. 1984. Estudo sobre o crescimento de Penaeus (Farfantepenaeus) paulensis Pérez Farfante, 1967 da Lagoa dos Patos, RS, Brasil (Decapoda, Penaeidae). Atlântica, Rio Grande, 7: 73-84.

Fransozo, A.; R.C. Costa; M.A.A. Pinheiro; S. Santos \& F.L.M. Mantelatto. 2000. Juvenile recruitment of seabob Xiphopenaeus kroyeri (Heller, 1862) (Decapoda, Penaeidea) in the Fortaleza Bay, Ubatuba, SP, Brazil. Nauplis, Rio Grande, 8 (2): $179-184$.

Gep/Cttmar. 2000. Boletim estatístico da pesca industrial de Santa Catarina. Ações Prioritárias ao desenvolvimento da pesca e aqüicultura no Sul do Brasil. Itajaí, UNIVALI, 61p.

Graça-Lopes, R.; A. Puzzi; E. Severino-Rodrigues; A.S. Bartolotto; D.S.F. Guerra \& K.T.B. Figueiredo. 2002. Comparação entre a produção de camarão sete-barbas e de fauna acompanhante pela frota de pequeno porte sediada na Praia de Perequê, Estado de São Paulo, Brasil. Boletim do Instituto de Pesca, São Paulo, 28 (2): 189-194.

Hill, B.J. \& T.J. WASSENBERG. 2000. The probable fate of discards from prawn trawlers fishing near coral reefs. A study in the northern Great Barrier Reef, Australia. Fisheries Research, Sidney, 48: 277-286.

Lunardon-Branco, M.J. \& J.O. Branco. 1993. A fauna de Brachyura de Menticirrus littoralis (Holbrook, 1860) na região de Matinhos e Caiobá, litoral do Paraná, Brasil. Arqui-

Revista Brasileira de Zoologia 21 (2): 295-301, junho 2004 
vos de Biologia e Tecnologia, Curitiba, 36 (3): 479-487.

Mantelatto, F.L.M. \& A. Fransozo. 2000. Brachyuran community in Ubatuba bay, northern coast of São Paulo state, Brazil. Journal of Shellfish Research, Maine, 19 (2): 701-709.

Mantelatto, F.L.M.; A. Fransozo \& M. L. Negreiros-Fransozo. 1995. Populational structure of Hepatus pudibundus (HERBST, 1785) (Decapoda, Brachyura, Calappidae) Fortaleza bay, Ubatuba, SP, Brasil. Revista de Biologia Tropical, San José, 43 (1): 259-264.

MatsuURA, Y. 1986. Contribuição ao estudo da estrutura oceanográfica da região Sudeste entre Cabo Frio (RJ) e Cabo de Santa Marta (SC). Ciência Cultura, Rio de Janeiro, 38 (8):1439-1450.

Melo, G.A.S. 1996. Manual de identificação dos Brachyura (caranguejos e siris) do litoral brasileiro. São Paulo, Plêiade/FAPESP, 604p.

Mota-Alves, M.I. \& M.M. Rodrigues. 1977. Aspectos da reprodução do camarão sete-barbas Xiphopenaeus kroyeri (Heller,1862) (Decapoda, Macrura), na costa do estado do Ceará. Arquivo de Ciência Marinha, Ceará, 17 (1): 29-35.

Nagakaki, J.M. \& M.L. Negreiros-Fransozo. 1998. Population biology of Xiphopenaeus kroyeri (Decapoda: Penaeidae) from Ubatuba Bay, São Paulo, Brazil. Journal of Shellfish Research, Maine, 17 (4): 931-935.

Nagakaki, J.M.; M.L. Negreiros-Fransozo \& A. Fransozo. 1995. Composição e abundância de camarões marinhos (Crustacea, Decapoda, Penaeidae) na Enseada de Ubatuba, Ubatuba (SP), Brasil. Arquivos de Biologia e Tecnologia, Curitiba, 38 (2): 583-591.

Nascimento, P.A.M. \& C.R. Poli. 1986. Curva de crescimento do camarão sete-barbas, Xiphopenaeus kroyeri (Heller,1862), na Baía de Tijucas, Santa Catarina, In: Anais do I Seminário sobre Ciências do Mar da UFSC, Florianópolis, 1: 37-41.

NeIva, G.S. \& J.P. WISE. 1963. The biology and fishery of the sea bob shrimp of Santos Bay, Brazil. Process of Gulf Caribbean Fisheries Institute, Miami, 16: 131-139.

Oshiro, L.M.Y.; R. SiLVA \& C.M. SiLVeIRA. 1999. Rendimento de carne nos caranguejos guaiá, Menippe nodifrons STIMPSON, 1859 e guaiamum, Cardisoma guanhumi Latreille, 1852 (Crustacea, Decapoda, Brachyura) da baía de Sepetiba/RJ. Acta Biologia Leopoldensia, São Leopoldo, 21 (1): 83-88.

Pereira-Barros, J.B. 1981. Sobre a ocorrência de siris do gênero Callinectes em Alagoas. Boletim Nível Estudos Ciências Marinhas, Maceió, 5: 1-24.

Pereira-Barros, J.B. \& I.B. Travassos. 1972. Informes sobre a pesca e biologia do siri tinga (Callinectes danae) e guajaú (Callinectes bocourti), na Lagoa Mundaú - Maceió - Alagoas. Maceió, Universidade Federal de Alagoas, parte 2, 518p.

Perez-Farfante, I. 1978. Srhimps and prawns. In: Fisher, W. (Ed.). FAO species identifications sheets for fishery proposes. Western Central Atlantic (Fishery Area 31), Rome, FAO, vol. 6, 40p.

PetTi, M.A.V. 1997. Papel dos crustáceos braquiúros na rede trófica da plataforma interna de Ubatuba, São Paulo (Brasil). Nerítica, Curitiba, 11: 123-137.

Pires-Vanin, A.M.S.; J.P.S. Jorge \& S. SARTor. 1995. Variação diária e sazonal da fauna bêntica da plataforma continental no litoral norte do Estado de São Paulo. Publicação especial do Instituto Oceanográfico, São Paulo, 35 (1):1-5.

Roman-COntreras, R. 1986. Análisis de la población de Callinectes spp. (Decapoda: Portunidae)en el sector occidental de la Laguna de Terminos, Campeche, México. Anais Instituto Ciencia del Mar y Limnologia, México, 13 (1): 315-322.

Ruffino, M.L. \& J. P. CAstello. 1992. Alterações na ictiofauna acompanhante da pesca do camarão-barba-ruça (Artemesia longinaris) nas imediações da Barra de Rio Grande, Rio Grande do Sul - Brasil. Nerítica, Curitiba, 7 (1-2): 43-55.

Sampaio, C.M.S. \& J. Fausto-Filho. 1984. Considerações sobre a bioecologia dos crustáceos decápodes da enseada do Macuripe (Fortaleza, Ceará, Brasil). Arquivo Ciência Marinha, Fortaleza, 23: 11-24.

Santos, E.P.; G.S. Neiva \& Y. Schaeffer. 1969. Dinâmica da população do camarão sete-barbas, Xiphopenaeus kroyeri (Heller, 1862) na baía de Santos. Pescarias Pesquisas, São Paulo, 2 (2): 41-55.

Santos, M.C.F. \& P.A. Coelho. 1996. Estudo sobre Xiphopenaeus kroyeri (Heller, 1862) (Crustácea, Decapoda, Penaeidae) em Luís Correia, PI. Trabalhos Oceanográficos Universidade Federal de Pernambuco, Recife, 24: 241-248.

Santos, S.; M.L. Negreiros-Fransozo, \& A. Fransozo. 2000. The distribution of the swimming crab Portunus spinimanus Latreille, 1819 (Crustacea, Brachyura, Portunidae) in Fortaleza bay, Ubatuba, SP, Brasil. Atlântica, Rio Grande, 16: 125-141.

Severino-Rodrigues, E.; J.B. Pita; R. Graça-Lopes; J.A.P. Coelho \& A. Puzzi. 1992. Aspectos biológicos e pesqueiros do camarão sete-barbas (Xiphopenaeus kroyeri) capturado pela pesca artesanal nolitoral do estado de São Paulo. Boletim do Instituto de Pesca, São Paulo, 19 (1): 67-81.

Severino-Rodrigues, E.; R. Graça-Lopes; J.B. Pita \& J.A.P. Coelho. 1985. Levantamento das espécies de camarão presentes no produto da pesca dirigida ao camarão sete-barbas (Xiphopenaeus kroyeri Heller, 1862) no estado de São Paulo, Brasil. Boletim do Instituto de Pesca, São Paulo, 12 (4): 77-85.

Severino-Rodrigues, E.; D.S.F. Guerra \& R. Graça-Lopes. 2002. Carcinofauna acompanhante da pesca dirigida ao camarão sete-barbas (Xiphopenaeus kroyeri) desembarcada na Praia do Perequê, Estado de São Paulo, Brasil. Boletim do Instituto da Pesca, São Paulo, 28 (1): 33-48.

SOKAL, R.R. \& F.J. RoHLF. 1969. Biometry, the principles and practices of statistics in biological research. San Francisco, W.H. Freeman, 776p.

Vieira, B.B. 1947. Observações sobre a maturação de Xiphopenaeus kroyeri no litoral de São Paulo. Boletim Museu Nacional, Rio de Janeiro, 74: 1-22.

Recebido em 05.XII.2003; aceito em 31.V.2004. 